\title{
Obesity is not associated with contrast nephropathy
}

This article was published in the following Dove Press journal:

Therapeutics and Clinical Risk Management

2 May 2010

Number of times this article has been viewed

\author{
Navin Jaipaul' \\ Rendell Manalo² \\ Seyed-Ali Sadjadi' \\ James McMillan' \\ 'Section of Nephrology, VA Loma \\ Linda Healthcare System, \\ ${ }^{2}$ Department of Medicine, Loma \\ Linda University School of Medicine, \\ Loma Linda, CA, USA
}

Background: Exposure to radiocontrast media may result in acute kidney injury (AKI) or traditionally defined contrast nephropathy $(\mathrm{CN})$, both of which may lead to increased morbidity and mortality. The pathogenesis of both these variants of contrast-induced nephropathy (CIN) may involve inflammatory mediators that lead to renal impairment. A link between obesity and inflammation has been clearly established, but whether obesity is independently associated with CIN is unknown.

Objective: To determine whether obesity, when stratified by body mass index (BMI), is a risk factor for CIN in a large and hemodynamically stable population of hospitalized United States veterans.

Design: Retrospective chart review.

Measurements: Presence or absence of AKI or $\mathrm{CN}$ after intravenous radiocontrast administration and comparison of patient characteristics between those with versus without AKI or CN.

Results: The overall prevalence of AKI and $\mathrm{CN}$ was $16.1 \%$ and $12.6 \%$, respectively. Patients with $\mathrm{AKI}$ or $\mathrm{CN}$ were comparable to those without radiocontrast injury, except that affected patients tended to be older and diabetic. When stratified by BMI, obesity was not found to be associated with the development of AKI or $\mathrm{CN}$ after exposure to radiocontrast.

Conclusion: Obesity does not appear to be an independent risk factor for AKI or CN after exposure to radiocontrast.

Keywords: obesity, contrast nephropathy, kidney injury

\section{Introduction}

Administration of intravenous radiocontrast material may result in either acute kidney injury or traditionally defined contrast nephropathy, usually within the first 24-72 hours after the contrast is administered. Acute kidney injury has been defined as an abrupt (within 72 hours) reduction in kidney function or an absolute increase in serum creatinine of $>0.3 \mathrm{mg} / \mathrm{dL}$, compared with traditional contrast nephropathy which has been defined in the same context as an absolute increase in the serum creatinine of $>0.5 \mathrm{mg} / \mathrm{dL} .{ }^{1}$ Either type of kidney injury due to contrast may be associated with increased morbidity and mortality, in particular, as the risk of contrast-induced nephropathy (CIN) increases with the number of risk factors (Note: The term contrast-induced nephropathy used here includes both contrast-induced acute kidney injury and traditional contrast nephropathy). The pathogenesis of CIN may be mediated by various inflammatory-related factors that involve the ionic and viscous composition of the contrast itself, renal vasoconstriction, and direct tubular injury. ${ }^{2-8}$
Correspondence: Navin Jaipaul

VA Loma Linda Healthcare System,

I I 20 I Benton St (I I IN), Loma

Linda, CA 92357, USA

Tel +l 9095836090

Fax + I 9097773858

Email navin.jaipaul@va.gov 
The main risk factors for CIN that have been reported in the literature include chronic kidney disease (stage III or greater; estimated glomerular filtration rate [eGFR] $<60 \mathrm{~mL} / \mathrm{min} / 1.73 \mathrm{~m}^{2}$ ), diabetes mellitus, volume depletion, nephrotoxic drug use (nonsteroidal anti-inflammatory drugs, cyclosporine, aminoglycosides), preprocedural hemodynamic instability, and other comorbidities (anemia, congestive heart failure, and hypoalbuminemia). ${ }^{9}$

Additionally, obesity may be associated with insulin resistance in both nondiabetic and diabetic patients, atherosclerosis, and the sequela of nonalcoholic fatty liver disease. ${ }^{10}$ Subacute and chronic inflammation often accompanies these obesity-related states, with elevated circulating levels of inflammatory markers. ${ }^{11,12}$ For example, F2-isoprostanes are prostaglandin-like compounds that serve as markers of oxidative injury, being generated from the free-radical initiated peroxidation of arachidonic acid. ${ }^{13}$ Elevated levels of F2-isoprostanes have been reported in both patients with obesity ${ }^{14}$ and CIN.${ }^{15}$ It is biologically plausible, therefore, that obesity may be independently associated with CIN, linked via a common inflammatory pathway. However, there is a paucity of data in the existing literature investigating whether obesity may be an additional independent risk factor for CIN. We therefore sought to determine whether obesity may be associated with CIN among a large cohort of hospitalized United States veterans.

\section{Methods}

\section{Study sample}

We conducted a retrospective chart review of hospitalized patients at a single Veterans' Affairs (VA) medical center in the southwestern United States. The Institutional Review Board at the medical center approved the study. By computerized data extraction, we identified all patients hospitalized between October 1, 2005, and September 30, 2006, who received intravenous radiocontrast material (identified by Current Procedural Terminology [CPT] coding) and had one or more serum creatinine values within 24 hours preprocedure and 72 hours postprocedure. Patients undergoing cardiac catheterization or on dialysis were excluded. Using these criteria, 803 patients were ultimately included in the study. The following additional demographic and comorbidity patient variables were extracted by computerized review: age, gender, race, body mass index (BMI), and the presence or absence of diabetes mellitus, congestive heart failure, liver disease, coronary artery disease, peripheral vascular disease, and smoking. Comorbidities were identified by International Classification of Disease, Ninth Revision codes. To indirectly measure the preprocedure volume status of our patients, we also extracted computerized data about the blood urea nitrogen (BUN) level (used in calculating the BUN/creatinine ratio) and systolic blood pressure on the day of the procedure, prior to the contrast administration.

\section{Intravenous contrast administration}

At the time of this study, our medical center did not have a formal protocol in place for preprocedure assessment of patients receiving intravenous contrast. Generally, the referring physician would determine the need for periprocedure hydration with isotonic saline or use of $\mathrm{N}$-acetylcysteine. However, all patients were administered nonionic, iso-osmolal contrast agents. The majority of patients were admitted to the hospital for a nonsepsis condition and underwent routine computed tomography (CT) of the chest, abdomen, and/or pelvis, or CT angiographic studies of the coronary, pulmonary, abdominal, or lower extremity vasculature.

\section{Measurements}

Two trained reviewers used the VA electronic medical record to identify the date of the procedure involving intravenous radiocontrast administration and abstract the most recent serum creatinine value, usually the day before the procedure (baseline level), and highest serum creatinine value within 72 hours after the procedure (peak level). Using the predefined criteria for acute kidney injury and traditional contrast nephropathy previously mentioned, these primary outcomes were dichotomized as present or absent according to the absolute change between peak and baseline serum creatinine values. Obesity was categorized according to stage of BMI: Stage 0, Underweight, BMI < 20; Stage 1, Normal, BMI < 25; Stage 2, Overweight, BMI 25-30; Stage 3, Obese, BMI 31-35; Stage 4, Morbidly Obese, BMI > 35.

\section{Statistical power calculation}

Using a conservative $3 \%$ estimate for the difference in the prevalence rate of contrast-induced nephropathy between obese and nonobese individuals (13\% obese vs $10 \%$ nonobese), and a prespecified alpha level of 0.05 , we determined a minimal needed sample size of 146 patients in each group of this population subset in order to achieve a power of $80 \%$.

\section{Statistical analysis}

Clinical and demographic characteristics were compared between patients with or without contrast-induced 
Table I Comparison of patients with versus without $\mathrm{CN}$ or $\mathrm{AKI}$ after intravenous contrast exposure

\begin{tabular}{|c|c|c|c|c|c|c|}
\hline Characteristic & $\begin{array}{l}\text { No } C N \\
(N=702)\end{array}$ & $\begin{array}{l}C N \\
(N=\mid 0 I)\end{array}$ & $P$ value & $\begin{array}{l}\text { No AKI } \\
(N=674)\end{array}$ & $\begin{array}{l}\text { AKI } \\
(N=129)\end{array}$ & $P$ value \\
\hline Age, years & $64.6( \pm 13.1)$ & $67.1( \pm 12.8)$ & 0.07 & $64.5( \pm 12.9)$ & $67.1( \pm 12.7)$ & 0.04 \\
\hline Male gender, $\mathrm{n}$ & 667 & 96 & 0.99 & 639 & 124 & 0.53 \\
\hline White race, $n$ & 564 & 84 & 0.67 & 541 & 107 & 0.42 \\
\hline Diabetes mellitus, $\mathrm{n}$ & 218 & 42 & 0.03 & 209 & 51 & 0.06 \\
\hline Heart failure, $n$ & 98 & 15 & 0.81 & 92 & 21 & 0.43 \\
\hline Liver disease, $\mathrm{n}$ & 59 & 5 & 0.23 & 58 & 6 & 0.13 \\
\hline Coronary artery disease, $n$ & 95 & 18 & 0.25 & 90 & 23 & 0.18 \\
\hline Peripheral vascular disease, $n$ & 176 & 26 & 0.88 & 164 & 38 & 0.22 \\
\hline Smoking, $\mathrm{n}$ & 29 & 5 & 0.70 & 28 & 6 & 0.80 \\
\hline $\mathrm{BMI}, \mathrm{kg} / \mathrm{m}^{2}$ & $27.3( \pm 6.9)$ & $27.4( \pm 6.6)$ & 0.88 & $27.3( \pm 7.0)$ & $27.6( \pm 6.5)$ & 0.64 \\
\hline Obesity Staging, $n$ & & & 0.93 & & & 0.88 \\
\hline Stage 0 (BMI < 20) Underweight & 78 & 9 & 0.49 & 76 & II & 0.29 \\
\hline Stage I (BMI 20-25) Normal & 208 & 30 & 0.86 & 201 & 37 & 0.98 \\
\hline Stage 2 (BMI 25-30) Overweight & 211 & 33 & 0.28 & 203 & 41 & 0.21 \\
\hline Stage 3 (BMI 3I-35) Obese & 128 & 17 & 0.26 & 120 & 25 & 0.05 \\
\hline Stage $4(\mathrm{BMI}>35)$ Morbidly Obese & 77 & 12 & 0.95 & 74 & 15 & 0.96 \\
\hline Systolic blood pressure, $\mathrm{mmHg}$ & $124.7( \pm 20.5)$ & $126.1( \pm 24.8)$ & 0.56 & $124.7( \pm 20.5)$ & $125.7( \pm 23.7)$ & 0.63 \\
\hline Blood urea nitrogen/creatinine ratio & $15.5( \pm 8.3)$ & I7.I ( $( \pm 8.7)$ & 0.07 & $15.4( \pm 8.2)$ & $17.2( \pm 8.9)$ & 0.07 \\
\hline Baseline creatinine, $\mathrm{mg} / \mathrm{dL}$ & $1.0( \pm 0.3)$ & $1.0( \pm 0.3)$ & 0.08 & $\mathrm{I} .0( \pm 0.3)$ & $1.0( \pm 0.3)$ & 0.67 \\
\hline Peak creatinine, ${ }^{*} \mathrm{mg} / \mathrm{dL}$ & $1.0( \pm 0.3)$ & $\mathrm{I} .4( \pm 0.5)$ & $<0.01$ & $1.0( \pm 0.3)$ & $1.4( \pm 0.5)$ & $<0.01$ \\
\hline
\end{tabular}

Notes: "Within 72 hours postcontrast.

Abbreviations: AKI, acute kidney injury; BMI, body mass index; $\mathrm{CN}$, contrast nephropathy.

acute kidney injury or traditional contrast nephropathy, using $\chi^{2}$ tests for dichotomous variables and $t$-tests for continuous variables. Factors significantly associated with contrast-induced nephropathy in bivariate analysis were entered in logistic regression models to determine independent predictors of contrast nephropathy. We considered $P$ values $<0.05$ to be significant. Inter-rater agreement on the primary outcomes of interests between the reviewers was assessed using the $k$ statistic on a $10 \%$ subset. All statistical analysis was performed with the SPSS statistical package (version 17.0; SPSS Inc., Chicago, IL).

\section{Results}

The baseline characteristics of our study population are shown in Table 1 . In this predominantly Caucasian male study population, diabetes was the most common comorbid condition, affecting $32 \%$ of the population. Other comorbidities examined included heart failure (14\%), liver disease $(8 \%)$, coronary artery disease $(14 \%)$, peripheral vascular disease $(25 \%)$, and cigarette smoking $(4 \%)$. The majority of the patients were overweight with an average BMI of 27.3. Approximately $14 \%$ of patients were obese (BMI 31-35), while 9\% were morbidly obese (BMI > 35). A minority of patients were underweight $(\mathrm{BMI}<20)$. Our study population represented a hemodynamically stable cohort of patients regardless of the presence or absence of
$\mathrm{CIN}$, as evidenced by a mean $\mathrm{BUN} /$ creatinine ratio below 20 and mean systolic blood pressure above $120 \mathrm{mmHg}$ across all groups studied.

The overall prevalence of acute kidney injury (AKI) and traditional contrast nephropathy $(\mathrm{CN})$ was $16.1 \%$ and $12.6 \%$, respectively. Patients with AKI or $\mathrm{CN}$ were comparable to those without radiocontrast injury, except that affected patients tended to be older and diabetic, $P<0.05$ (Table 1). Specifically, each additional year of age was associated with a statistically significant $2 \%$ increase in the odds of AKI (odds ratio $[\mathrm{OR}]=1.02 ; 95 \%$ confidence interval $[\mathrm{CI}]: 1.01-1.03)$, and the presence of diabetes was associated with a statistically significant $56 \%$ increased odds of $\mathrm{CN}(\mathrm{OR}=1.56 ; 95 \%$ CI: 1.01-2.39) (Table 2). When stratified by BMI, obesity was not found to be associated with the development of AKI or $\mathrm{CN}$ after exposure to radiocontrast (Table 1). Being

Table 2 Logistic regression models for odds ratio estimates of $\mathrm{CN}$ or $\mathrm{AKI}$ associated with age and diabetes mellitus

\begin{tabular}{|c|c|c|c|c|c|c|}
\hline \multirow[t]{3}{*}{ Variable } & \multicolumn{3}{|l|}{$\mathrm{CN}$} & \multicolumn{3}{|l|}{ AKI } \\
\hline & \multicolumn{3}{|c|}{$95 \% \mathrm{Cl}$} & \multicolumn{3}{|c|}{$95 \% \mathrm{Cl}$} \\
\hline & OR & Lower & Upper & OR & Lower & Upper \\
\hline Age & 1.01 & 1.00 & 1.03 & 1.02 & 1.01 & 1.03 \\
\hline Diabetes & 1.56 & 1.01 & 2.39 & 1.43 & 1.00 & 2.11 \\
\hline
\end{tabular}

Abbreviations: $\mathrm{AKI}$, acute kidney injury; $\mathrm{Cl}$, confidence interval; $\mathrm{CN}$, contrast nephropathy; OR, odds ratio. 
underweight was also not associated with the development of AKI or CN after contrast exposure (Table 1). Complete agreement between the two reviewers regarding the development of AKI or CN was substantial (98\%).

\section{Discussion}

The focus of this study was to determine whether obesity, when stratified by BMI, is a risk factor for CIN in a large and hemodynamically stable population of hospitalized United States veterans. For this reason, we purposefully excluded patients undergoing cardiac catheterization because of increased risk of hemodynamic instability in this subset of hospitalized patients. Hemodynamic alterations in these patients would predispose to other forms of hypoperfusioninduced kidney injury that would be indistinguishable from contrast-induced nephropathy by the defined criteria. Despite exclusion of these patients, our study was still more than adequately powered to detect even a conservative estimate for the difference in the prevalence rate of contrast-induced nephropathy between obese and nonobese individuals, as noted in the Methods section.

The prevalence rates of $\mathrm{AKI}$ and $\mathrm{CN}$ observed in this study are consistent with those published previously in the literature. ${ }^{1}$ In addition, we found that older age and the presence of diabetes are significantly associated with the development of CIN. These are also known associations that have been previously reported in the literature, ${ }^{1}$ and we verified their independent association in the direction and magnitude expected based on logistic regression models (Table 2). In our study, however, obesity was not found to be independently associated with the development of CIN. This lack of a relationship between obesity and CIN persisted regardless of stage of obesity or level of kidney function. Despite the biological plausibility of an inflammatory role linking obesity to CIN, the absence of an observed association suggests that increased tissue adiposity alone is not sufficient to increase the risk of CIN, or that other unaccounted factors may mitigate the effects of increased tissue adiposity on the kidney after contrast exposure. For instance, obesity is known to induce glomerular hyperfiltration which may enhance tubular sodium reabsorption and contribute to a transiently volume expanded state. ${ }^{16}$ Plausibly, therefore, such a volume expanded state might reduce the risk of CIN. It is also well known that GFR estimated by the Modification of Diet in Renal Disease (MDRD) formula tends to underestimate actual GFR in obese individuals, and therefore, it is possible that some of our patients had higher GFRs and were at lower risk for the development of CIN. ${ }^{17,18}$ Along this line of thought, however, it is important to point out that we neither observed an increased risk of obesity with CIN, nor a protective effect of obesity on CIN. Similarly, we observed no association of being underweight with the risk of CIN, even though underweight individuals may have diminished physiologic reserve.

It is also interesting to note that in this study, obesity was not a surrogate marker of diabetes as may be expected. Nevertheless, we believe that the results of our study are valid due to the fact that we observed prevalence rates of CIN and expected associations of known variables with CIN as previously mentioned.

Limitations of this study include its observational nature in a single medical center and lack of generalizability to other populations. Additionally, we did not have access to information about concurrent medication use such as the dose of contrast given, or statin therapy which is known to have anti-inflammatory properties. ${ }^{19}$ However, the potential anti-inflammatory effects of statin therapy on the kidney, and specifically CIN, remain still largely speculative and unproven. In fact, recent observational data suggests that statin usage prior to primary percutaneous coronary intervention is not associated with reduction in CIN. ${ }^{20}$ Similarly, the benefit of periprocedure treatment with $\mathrm{N}$-acetylcysteine in reducing $\mathrm{CIN}$ is unproven, ${ }^{21}$ and we did not have access to information about its use among our study population. We were also unable to gauge directly the volume status of the patients included in the study. However, we evaluated reasonable surrogate markers for preprocedure volume status such as BUN/creatinine ratio and systolic blood pressure. These measurements suggested that our study population was adequately volume replete and hemodynamically stable prior to the administration of intravenous contrast.

Despite the aforementioned limitations of our study, this particular population of patients is representative of a typical cohort at risk for CIN, and our finding that obesity is not an independent risk factor for contrast nephropathy should reassure medical providers of no added risk for kidney injury after intravenous contrast administration due to obesity alone.

\section{Acknowledgments}

This research was presented in poster abstract form at the 2009 American Society of Nephrology Meeting in San Diego, CA, USA. We would like to thank Ms Gloria Dowdy, Information Specialist, for her invaluable assistance with the computerized data extraction for this study. 


\section{Disclosures}

The authors report no conflicts of interest in this work.

\section{References}

1. Lautin EM, Freeman NJ, Schoenfeld AH, et al. Radiocontrast-associated renal dysfunction: incidence and risk factors. Am J Roentgenol. 1991;157:49-58.

2. Detrenis S, Meschi M, Musini S, Savazzi G. Lights and shadows on the pathogenesis of contrast-induced nephropathy: state of the art. Nephrol Dial Transplant. 2005;20:1542-1550.

3. Persson PB, Hansell P, Liss P. Pathophysiology of contrast mediuminduced nephropathy. Kidney Int. 2005;68:14-22.

4. Heyman SN, Rosenberger C, Rosen S. Regional alterations in renal haemodynamics and oxygenation: a role in contrast medium-induced nephropathy. Nephrol Dial Transplant. 2005;(20 Suppl 1):i6-i11.

5. Agmon Y, Peleg H, Greenfield Z, Rosen S, Brezis M. Nitric oxide and prostanoids protect the renal outer medulla from radiocontrast toxicity in the rat. J Clin Invest. 1994;94:1069-1075.

6. Weisberg LS, Kurnik PB, Kurnik BR. Radiocontrast-induced nephropathy in humans. Role of renal vasoconstriction. Kidney Int. 1992;41:1408-1415.

7. Cantley LG, Spokes K, Clark BA, McMahon EG, Carter J, Epstein FH. Role of endothelin and prostaglandins in radiocontrast-induced renal artery constriction. Kidney Int. 1993;44:1217-1223.

8. Russo D, Minutolo R, Cianciaruso B, Memoli B, Conte G, De Nicola L. Early effects of contrast media on renal hemodynamics and tubular function in chronic renal failure. J Am Soc Nephrol. 1995;6:1451-1458.

9. McCullough PA, Adam A, Becker CR, et al. Risk prediction of contrast-induced nephropathy. Am J Cardiol. 2006;98(6A):27K-36K.

10. Shoelson SE, Herrero L, Naaz A. Obesity, inflammation, and insulin resistance. Gastroenterology. 2007;132:2169-2180.

11. Knight SF, Imig JD. Obesity, insulin resistance, and renal function. Microcirculation. 2007;14(4-5):349-362.
12. Calabro P, Yeh ET. Obesity, inflammation, and vascular disease: the role of the adipose tissue as an endocrine organ. Subcell Biochem. 2007;42:63-91.

13. Milne GL, Musiek ES, Morrow JD. F2-isoprostanes as markers of oxidative stress in vivo: an overview. Biomarkers. 2005; Suppl 1: S10-S23.

14. Dietrich M, Block G, Hudes M, et al. Antioxidant supplementation decreases lipid peroxidation biomarker F2-isoprostanes in plasma of smokers. Cancer Epidemiol Biomarkers Prev. 2002;11:7-13.

15. Drager L, Andrade L, Barros de Toledo J, et al. Renal effects of $\mathrm{N}$-acetylcysteine in patients at risk for contrast nephropathy: decrease in oxidant stress-mediated renal tubular injury. Nephrol Dial Transplant. 2004;19:1803-1807.

16. Chagnac A, Herman M, Zingerman B, et al. Obesity-induced glomerular hyperfiltration: its involvement in the pathogenesis of tubular sodium reabsorption. Nephrol Dial Transplant. 2008;23(12):3946-3952.

17. Cirillo M, Anastasio P, De Santo NG. Relationship of gender, age and body mass index to errors in predicted kidney function. Nephrol Dial Transplant. 2005;20:1791-1798.

18. Verhave JC, Fesler P, Ribstein J, Cailar G, Mimran A. Estimation of renal function in subjects with normal serum creatinine levels: influence of age and body mass index. Am J Kidney Dis. 2005;46:233-241.

19. Blanco-Colio L, Tunon J, Martin-Ventura J, Egido J. Anti-inflammatory and immunomodulatory effects of statins. Kindey Int. 2003;63: 12-23.

20. Bouzas-Mosquera A, Vazquez-Rodriguez JM, Calvino-Santos R, Vázquez-González N, Castro-Beiras A. Statin therapy and contrast-induced nephropathy after primary angioplasty. Int J Cardiol. 2009;134:430-431.

21. Gonzales DA, Norsworthy KJ, Kern SJ, et al. A meta-analysis of $\mathrm{N}$-acetylcysteine in contrast-induced nephropathy: unsupervised clustering to resolve heterogeneity. BMC Med. 2007;5:32.
Therapeutics and Clinical Risk Management

\section{Publish your work in this journal}

Therapeutics and Clinical Risk Management is an international, peerreviewed journal of clinical therapeutics and risk management, focusing on concise rapid reporting of clinical studies in all therapeutic areas, outcomes, safety, and programs for the effective, safe, and sustained use of medicines. This journal is indexed on PubMed Central, CAS,

\section{Dovepress}

EMBase, Scopus and the Elsevier Bibliographic databases. The manuscript management system is completely online and includes a very quick and fair peer-review system, which is all easy to use. Visit http://www.dovepress.com/testimonials.php to read real quotes from published authors. 